\title{
Robotic exoskeletons: a perspective for the rehabilitation of arm coordination in stroke patients
}

\author{
Nathanaël Jarrassé ${ }^{1,2,3}$,Tommaso Proietti ${ }^{1,2,3}$, Vincent Crocher ${ }^{1,2,4}$, Johanna Robertson ${ }^{5}$, Anis Sahbani ${ }^{1,2,3}$, \\ Guillaume Morel ${ }^{1,2,3}$ and Agnès Roby-Brami ${ }^{1,2,3,5}$ *
}

UMR 7222, Center National de la Recherche Scientifique (CNRS), Institute of Intelligent Systems and Robotics (ISIR), Paris, France

2 UMR 7222, Sorbonne Universités, UPMC Univ Paris, Paris, France

${ }^{3}$ U1150, Institut National de la Santé et de la Recherche Médicale (INSERM), Agathe-ISIR, Paris, France

${ }^{4}$ Department of Electrical and Electronic Engineering, University of Melbourne, Melbourne, VIC, Australia

${ }^{5}$ Department of Physical Medicine and Rehabilitation, Hôpital Raymond Poincaré, Garches, France

\section{Edited by:}

Ana Bengoetxea, Universidad del País

Vasco-Euskal Herriko Unibertsitatea

(UPV/EHU), Spain

Reviewed by:

Renaud Ronsse, Catholic University of Louvain, Belgium

Federico Posteraro, Versilia Hospital, Italy

\section{${ }^{*}$ Correspondence}

Agnès Roby-Brami, UMR 7222

Center National de la Recherche

Scientifique (CNRS), Institute of

Intelligent Systems and Robotics

(ISIR), 4 Place Jussieu, Paris 75005 ,

France

e-mail: roby-brami@isir.upmc.fr
Upper-limb impairment after stroke is caused by weakness, loss of individual joint control, spasticity, and abnormal synergies. Upper-limb movement frequently involves abnormal, stereotyped, and fixed synergies, likely related to the increased use of sub-cortical networks following the stroke. The flexible coordination of the shoulder and elbow joints is also disrupted. New methods for motor learning, based on the stimulation of activitydependent neural plasticity have been developed. These include robots that can adaptively assist active movements and generate many movement repetitions. However, most of these robots only control the movement of the hand in space. The aim of the present text is to analyze the potential of robotic exoskeletons to specifically rehabilitate joint motion and particularly inter-joint coordination. First, a review of studies on upper-limb coordination in stroke patients is presented and the potential for recovery of coordination is examined. Second, issues relating to the mechanical design of exoskeletons and the transmission of constraints between the robotic and human limbs are discussed. The third section considers the development of different methods to control exoskeletons: existing rehabilitation devices and approaches to the control and rehabilitation of joint coordinations are then reviewed, along with preliminary clinical results available. Finally, perspectives and future strategies for the design of control mechanisms for rehabilitation exoskeletons are discussed.

Keywords: rehabilitation robotics, exoskeleton, upper-limb, synergies, arm coordination control

\section{SHOULDER-ELBOW COORDINATION AND SYNERGIES IN STROKE PATIENTS}

Although stroke causes lesions of the motor areas of the brain, motor impairments occur in the body on the opposite side to the lesion (hemiplegia). During the weeks following the lesion, symptoms usually recover spontaneously but partially and inconstantly, and many patients are left with impairment of upper-limb movement (hemiparesis). The symptoms, which occur following a brain lesion, are classically termed negative symptoms (weakness (Colebatch and Gandevia, 1989; Sukal-Moulton et al., 2014), loss of individual joint control (Zackowski et al., 2004)) and positive symptoms with excessive muscle contractions (spasticity (Lance, 1980; Mottram et al., 2009), spastic co-contraction (Gracies, 2005), dystonia, or pathological synergies (Twitchell, 1951; Brunnstrom, 1970)). Together, the weakness and abnormal contractions result in a disruption of goal-directed upper-limb movements and hand dexterity, causing disability.

\subsection{SYNERGIES AND SHOULDER-ELBOW COORDINATION}

Different practical and theoretical approaches are used to describe shoulder-elbow coordination. These descriptions are not fully consistent, with the word "synergy" being used to describe different phenomena. On one hand, Bernstein (1967) described synergies as fundamental building blocks of motor control, which decrease the redundancy of the system. Indeed, the motor system has more degrees of freedom (DoF) than are necessary to carry out any task, for example, the articulated upper-limb has more than 7 degrees of freedom when only 6 are necessary for any grasping task. According to this concept, synergies (i) combine several elements, which share the same spatio-temporal properties and "work together," and (ii) may be combined in a task specific way so that a limited number of synergies can give rise to a continuum of responses. But, there is still no agreement on the space (muscle or joints) in which synergies are organized. For some authors, synergies are organized at the muscle level (Bizzi et al., 2008). Mathematical techniques such as linear decomposition have been used to identify muscle synergies in healthy subjects in a variety of tasks such as posture (Ting, 2007) or reaching (d'Avella et al., 2006, 2008). For other authors, synergies are organized at the joint level and are endowed with properties of flexibility and automatic compensation between elements in order to stabilize the important task related variable (Latash and Anson, 2006). On the other hand, the word synergy is also used to refer to the pathological coupling of movements observed in patients (Dewald and Beer, 2001). Clinical observations of the global and stereotyped patterns of movements that occur when stroke patients make any effort to move are described 
as pathological synergies. Couplings of shoulder elevation movements with elbow flexion (flexor synergy) or shoulder adduction/internal rotation with elbow extension (extensor synergy) have been reported (Brunnstrom, 1970). These abnormal synergies have been documented using quantitative experimental methods. Tasks involving the isometric generation of force have demonstrated that abnormal muscle coupling induces involuntary elbow flexion during voluntary shoulder abduction (Dewald et al., 1995; Dewald and Beer, 2001). During reaching movements in the horizontal plane at the shoulder level, target dependent perturbations of reaching kinematics and kinetics occur in stroke patients. These are reduced when the arm is supported against gravity (Beer et al., 2004; Sukal et al., 2007; Ellis et al., 2008). The available reaching workspace depends on the degree of shoulder loading (Sukal et al., 2007; Ellis et al., 2008). There are relatively few studies of muscle synergies using appropriate mathematical methods in hemiparetic patients. Cheung et al. (2009) showed that the structure of synergies was relatively preserved during various motor tasks in mildly affected patients but was either fractionated or merged in more impaired patients (Cheung et al., 2012). Using a 3D isometric task, Roh et al. (2013) did not find abnormal coupling between elbow and shoulder muscles but instead, abnormal and global activation of the three heads of the deltoid muscle during abduction.

Kinematic analysis of the time course of joint rotations showed a disruption of the relative timing between shoulder and elbow movements during reaching in stroke patients (Levin, 1996; Cirstea et al., 2003; van Kordelaar et al., 2012). During forward reaching movements, shoulder flexion and elbow extension tend to be reduced and shoulder abduction increased (Roby-Brami et al., 2003b). This elevates the elbow and alters the plane of arm movement (Merdler et al., 2013). The analysis of arm coordination is complicated by the fact that stroke patients develop compensatory trunk flexion (Cirstea and Levin, 2000; Roby-Brami et al., 2003a). A study based on principal component analysis (PCAs) showed that, when the trunk is fixed, patients with higher levels of impairment use fewer synergic joint combinations to carry out reaching tasks, suggesting that there is a reduction in the flexibility of synergies (Reisman and Scholz, 2003). Automatic error compensation between joint rotations is also impaired in stroke patients but appears to be task dependent since it is relatively preserved when the trunk is fixed (Reisman and Scholz, 2003) but not when trunk movement assists the reach (Reisman and Scholz, 2006). When the trunk is free, stroke patients use more synergic joint combinations than healthy subjects (van Kordelaar et al., 2012).

\subsection{MECHANISMS OF ALTERED JOINT COORDINATION AFTER STROKE}

The neurophysiology mechanisms behind synergies are still poorly understood. The specific role of the spinal cord (Bizzi et al., 2008), sub-cortical structures, and cortical areas (Cheney and Fetz, 1985; Capaday, 2004) in the generation of synergies is still unclear (i.e., in which structures of the hierarchical motor system are the elements gathered and the synergies combined?). Likewise, the relationship between voluntary control of individual joints, spasticity, and altered inter-joint coordination in stroke patients is still disputed. One current physiopathological hypothesis is that the abnormal fixed patterns are related to the activity of sub-cortical structures or networks. Owing to the alteration of the cortico-spinal command, the motor command is generated by less inhibited pathways, which originate in the brain-stem (Gracies, 2005; Cheung et al., 2009). This hypothesis is supported by the pattern of interaction of voluntary commands with multi-joint stretch reflexes (Trumbower et al., 2010), flexion reflexes (Dewald et al., 1999), the startle reflex (Honeycutt and Perreault, 2012), and neck rotation similar to the tonic neck reflex (Ellis et al., 2012). An increase in proprio-spinal relay is also likely (Mazevet et al., 2003). However, the alteration of the command, resulting from the lesion of the primary motor cortical area probably also has direct consequences on arm coordination. According to Zackowski et al. (2004), the main cause of impairment is the alteration of individual joint commands. Other authors have shown that reaching impairments are more related to a lack of recruitment of the agonists than to excessive coupling (Wagner et al., 2007; Prange et al., 2010). The abnormal coupling between joints could be a consequence of the distribution of muscle weakness and a saturation phenomenon (McCrea et al., 2005) (but this is contested by Beer et al. (2007)). The link between cortico-spinal command, spasticity, and coordination could be related to a deficit in the range of regulation of stretch reflex thresholds. The descending commands directly influence the level of excitability of the motoneuron membrane determining the position of the stretch reflex threshold relative to joint motion (Feldman et al., 2007). Impairments in the descending command modify its range of regulation causing spasticity (Levin and Dimov, 1997). Since spatial spasticity zones are modified by the shoulder-elbow configuration, this could be the basis of disordered upper-limb coordination (Musampa et al., 2007).

The mechanisms of recovery after stroke are multifactorial and the effect of rehabilitation programs is complex (Langhorne et al., 2011). Activity-dependent neural plasticity of the cortical maps adjacent to the lesion probably occurs, particularly during the acute period after stroke (Nudo, 2013). In order to stimulate such plasticity, many new rehabilitation methods, including robotic assistance, have been developed according to the principles of motor learning (Huang and Krakauer, 2009). In addition, the improvements measured by clinical scales can be due to the development of compensatory strategies. Compensatory strategies have an immediate benefit on daily life activity but, due to the learned disuse phenomenon, may have a negative impact on the quality of movement performance and limit the long-term prognosis (Taub et al., 2006; Levin et al., 2009). This is demonstrated by the possible benefits of constraint induced movement therapy (CIMT) and intensive task specific practice that can reduce learned disuse in the chronic stage of stroke (Wolf et al., 2006). After CIMT, some patients may continue to improve spontaneously if they have reached a given functional threshold so that they can use their limb spontaneously; if this is not the case, the benefit may be lost (Hidaka et al., 2012).

\subsection{ROBOTIC AND MECHANICAL ASSISTANCE FOR RECOVERY}

Numerous rehabilitation-robotic devices have been developed since the late 90s, particularly for the neurorehabilitation of post-stroke patients (see review in Brewer et al. (2007)). Most of these devices guide the movement of the hand in one plane. Some robots can passively mobilize the limb of patients with 
poor recovery or can provide precisely controlled active assistance as a function of patient's capacity. An advantage of robotic assistance is the possibility for patients to carry out a great number of movement repetitions, increasing the intensity of therapy. Recent extensive clinical testing of one of these devices, the InMotion ${ }^{\odot}$ robot (which has been used in clinical practice for many years) has demonstrated its effectiveness with significant improvements in arm motor capacity after a program of robot therapy. However, so far, there does not appear to be a qualitative benefit of robotic devices over a therapist performing the same quantity of movements (Volpe et al., 2008, 2009; Lo et al., 2010). Robot therapy still remains particularly interesting, however, since it affords more movement opportunities than standard therapy. Most previous clinical studies have been carried out with planar robotic manipulanda, which can only control the movement of the hand in space. In contrast, more conventional therapies (Brunnstrom, 1970; Bobath, 1990) particularly insist on the quality of the coordination based on the handling skills of the physical therapist. The physical therapist supports the weight of the upper limb by simultaneously holding the upper and the lower arm in order to mobilize the upper arm or to assist voluntary reaching movements. In addition, the support provided by the therapist is important for the prevention of shoulder-hand syndrome due to shoulder subluxation. A combination of these approaches would involve the insistence on the quality of coordination, monitored or guided by a therapist, while the patient practices a motor learning program (Levin and Panturin, 2011).

\subsection{CAN HEMIPARETIC PATIENTS RELEARN COORDINATION?}

The ability of stroke patients to retain or relearn upper-limb coordination remains a topic of debate. The improvement of arm coordination observed during the acute phase of stroke is attributed to a spontaneous recovery of individual muscle commands (van Kordelaar et al., 2013). However, experiments performed in the chronic phase of stroke suggest that hemiparetic patients might not use all their potential shoulder-elbow coordination if they have the possibility to compensate by using the trunk. When the trunk is restrained, patients with moderate impairment show an immediate improvement in shoulder-elbow joint range and coordination (Michaelsen et al., 2001). Further studies have shown that repetitive training of reaching increases the amount of elbow joint rotation when the trunk is restrained but increases trunk compensation if it is free (Michaelsen et al., 2006), see review in Wee et al. (2014). This is consistent with the observation that CIMT does not improve joint range of motion (Kitago et al., 2013) since CIMT allows trunk compensation. Training the upper limb in the horizontal plane with gravity compensation using a robotic manipulandum (Dipietro et al., 2007; Tropea et al., 2013), progressive abduction loading (Ellis et al., 2009), or antigravity support (Krabben et al., 2012) improves the volume of the available workspace and the smoothness of the movement, suggesting that an improvement of arm coordination or a reduction of abnormal coupling. Other studies have not confirmed the benefit of such repetitive training on joint range of motion (Frisoli et al., 2012). A recent study suggested slight modification of muscle synergies after treatment (Tropea et al., 2013). Evidence that training not only improves active joint range of motion but also inter-joint coordination is still lacking.

\section{DESIGN CHALLENGES FOR REHABILITATION EXOSKELETONS}

While upper-limb manipulanda and lower limb exoskeletons have been used in the clinical practice for several years now (see the manipulandum by InMotion ${ }^{\odot}$ and the Lokomat $^{\odot}$ by Hocoma), upper-limb exoskeletons have been only recently developed (mid 2000s) and their effects have been little studied. Indeed, the first commercially available upper-limb exoskeleton for rehabilitation was only released at the end of 2011 (Riener et al., 2011).

One major cause of this slow development is the complexity of the interaction between mechatronic structures and the human body, both at the physical and at the control level. While the pioneer devices only controlled hand motion in a single plane, exoskeletons provide 3D interaction at the joint level through their kinematic redundancy and the presence of multiple attachment points between the device and patient's limb. These characteristics offer new and interesting perspectives for rehabilitation, but make devices much more complex to design and control.

Moreover, one fundamental particularity of rehabilitation exoskeletons compared to exoskeletons, which are designed to assist fully paralyzed patients, is that they should be able to respond to any movement made by the patient (even pathological). This must be based on a fine control of the mechanical interaction with the patient's limb (Maclean et al., 2000): more than assisting the movement, the goal is to help the patient recover his/her sensorimotor capability. In order to be able to perform such a task, several challenges relating to the global mechanical design of these structures, their coupling with the human limb and, above all, their control, must be overcome.

\subsection{ANTAGONISTIC DESIGN: POWER VS BACKDRIVEABILITY}

From a mechanical point of view, an exoskeleton must be able to interact with the human body, a very complex kinematic structure. Exoskeletons must have a large number of active (motorized) joints, each with a wide range of motion to be able to follow as well as to assist movements within a large workspace. The exoskeleton must be able to both generate a high level of forces to sustain, assist, and/or perturb the motor capabilities of the patient, and to follow, without perturbing, human movements, which have large velocity and acceleration peaks, thus, requiring a high level of dynamic interaction. The main problem in designing such a device is the competing nature of these issues: a powerful exoskeleton requires large and heavy actuators; however, these limit angular displacement and therefore the workspace. Smaller, lighter actuators with gearboxes could generate sufficient forces, however, gearboxes add friction to the system, reducing overall dynamic performance. Innovative mechanical transmission combining high dynamics, forces, and ranges of velocities, which are compatible with human movement have been designed over the last 20 years, along with novel approaches to actuation (pneumatic, hydraulic, and cable actuators, combined, or not, with conventional electrical actuation) in order to adapt to the requirements for rehabilitation (Garrec et al., 2008). Thus, the challenge in the design of an exoskeleton is to achieve a 
“conceptual agreement" between power, workspace, dynamics, and weight.

\subsection{PHYSICAL COUPLING BETWEEN THE HUMAN BODY AND THE ROBOT AND KINEMATO-STATIC COMPATIBILITY}

For years, research has mainly focused on technological aspects (actuators, embedment, energy, etc.) and has followed the paradigm defined in Perry et al. (2007): "an exoskeleton is an external structural mechanism with joints and links corresponding to those of the human body." In other words, designing the kinematics of an exoskeleton generally consists of trying to replicate human limb kinematics. This has a number of advantages: similarity of the workspaces, avoidance of singularity (Pons, 2008), and oneto-one mapping of joint force capabilities over the workspace. However, this paradigm has a major disadvantage due to the fact that it is impossible to precisely replicate human kinematics with a robot. Indeed, there are two major problems: morphology varies drastically between subjects and joint kinematics are very complex and cannot be imitated by conventional robotic joints (Scott and Winter, 1993). Currently, there is no consensual model of human kinematics in the biomechanics literature due to the complex geometry of bone surfaces. For example, different models are used for the shoulder complex including the scapula and clavicle (Van der Helm et al., 1992). Discrepancies between the two kinematic chains thus seem unavoidable, generating kinematic incompatibility and thus hyperstaticity or "over-constraint": if the human and the robot are rigidly connected together through embedments at the different connection points, there will be more force and torque constraints than controlled mobility (active DoF) of the robot. The main consequence will be the occurrence of uncontrollable forces creating deformations of the subjects skin and tissues (Schiele, 2008) as can be seen in Figure 1A. This is even more problematic if the patient is fragile or has sensorimotor impairment.

Several approaches have been developed to avoid this problem. The first consists of designing an adaptable exoskeleton with adjustable length segments or the addition of redundant DoF serially in the robot kinematic chain to align active joint axes to the human joint axes (Housman et al., 2007). The other approach, based on the theory of mechanisms, involves the addition of passive DoF to connect the subject to the robot. Such a system allows the transmission of the robot-controlled forces while guaranteeing freedom of movement of the wearer and limiting the uncontrolled and undesired application of forces to his/her limb (Jarrassé and Morel, 2012; Galinski et al., 2013). An example of a fixation mechanism based on this approach is shown in Figure 1C: passive DoF fixations (each composed of a ball joint and a slider) are used to connect a human limb to a 4 active DoF ( 3 at the shoulder and 1 at the elbow) exoskeleton. Because of kinemato-static duality, the exoskeleton is only able to control 4 forces (shown in Figure 1B), which are the only forces, which are transmitted by these mechanisms (making the coupling isostatic). No torque (because of the rotation allowed by to the 3-DoF ball joint) or force (because of the $1 \mathrm{DoF}$ linear slider) can be applied along the limb segment. Moreover, the system guarantees kinematic compatibility between the exoskeleton and the wearer without requiring an accurate alignment of joint axes.

\subsection{TRANSPARENCY AND CONTROL SHARING}

The main objective of the research on the hardware design of exoskeletons is to maximize their interaction capacity.

Rehabilitation robotics began by using robots to passively mobilize patient's limbs during the first stages of rehabilitation (passive mode) when the patient is unable to move alone. However, the effectiveness of such passive movements for stimulating motor recovery was limited (Lynch et al., 2005). In order to stimulate motor recovery, it is essential for rehabilitation robots to exhibit finer mechanisms of interaction: shared control of movement must be possible as soon as the patient has recovered a minimal amount of motor capacity (Patton and Mussa-Ivaldi, 2002; Hogan et al., 2006). Indeed, since neurorehabilitation addresses issues related to motor learning, the machines must allow patients to express whatever movement they can without hindering or suppressing their motor capability (Hogan and Krebs, 2004).

Therefore, one key feature that rehabilitation exoskeletons should exhibit is transparency: the robot must be able to "hide" if the patient is capable of making the movement without assistance. In this situation (called active mode, as the termis based on the patient's activity), the robot is passive and thus must not perturb the patient's movement (especially since human movements
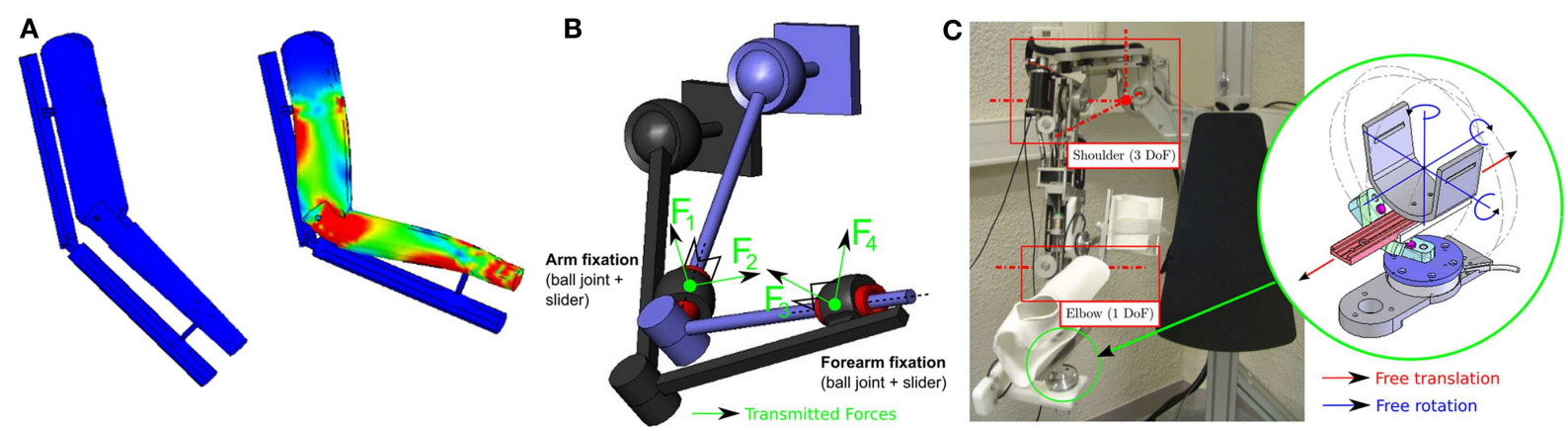

FIGURE 1 | (A) Visualization of the strain distribution on a human arm performing a flexion movement when it is rigidly connected to a simple $1 \mathrm{DoF}$ elbow exoskeleton with misaligned joint axes (Simulated with Solidworks ${ }^{\odot}$ ). Red areas represent the stress concentration zones. (B) Kinematic representation of a 4-DoF exoskeleton attached to a human arm (both with 3-DoF ball joint at the shoulder +1 pivot joint at the elbow) using passive joint fixations (Jarrassé and Morel, 2012). (C) Four active DoF exoskeleton (ABLE, see Garrec et al. (2008)) with its set of passive DoF fixations. 
are often performed with low limb stiffness, and are very sensitive to perturbations (Gomi and Kawato, 1997)). Equally, if the robot is used as a measurement tool to record the patient's movement, it must influence the movement as little as possible.

Despite efforts made to design backdrivable mechanical structures, most exoskeletons are not intrinsically transparent and rely on sensors and control algorithms to improve their interaction capacity and to make them behave "therapeutically."

\section{CONTROL OF EXOSKELETONS FOR UPPER-LIMB REHABILITATION}

\subsection{EXISTING ACTIVE EXOSKELETONS FOR REHABILITATION}

We carried out a review of active systems with 3-DoF or more (with a minimum of control on two upper-limb joints: shoulder, elbow, or wrist) and found 30 different exoskeleton prototypes for neurorehabilitation of the upper limbs. For this review, we searched PubMed, ClinicalTrials, IEEE Xplore Digital Library, Science Direct, and Google Scholar databases, using different combinations of the following keywords: "upper limb, robot, exoskeleton, rehabilitation, assisted, shoulder, elbow, wrist, arm, therapy, stroke, and training." Passive "spring-like" structures and cable robots with multiple contact points were not considered in this study. A few multi-contact multi-robot active systems were, however, included since they interact at the joint level similarly to exoskeletons (Lo and Xie, 2012; Maciejasz et al., 2014), see Table 1.

Most exoskeletons found were designed to affect either the shoulder and elbow joints or the shoulder, elbow, and wrist. To our knowledge, only 11 of these 30 devices have been tested on poststroke subjects, and few studies involved more than 10 post-stroke patients. About one-third of the devices found have not been the subject of publications in the main journals and conferences for at least 3 years.

\subsection{CHALLENGES IN THE CONTROL OF AN EXOSKELETON}

Existing controllers for exoskeletons are mostly assistive, according to the definition in Marchal-Crespo and Reinkensmeyer (2009): this involves replicating therapist behavior to assist the impaired subject to accomplish specific tasks. This paradigm can be implemented using different control techniques. The simplest way to control any robotic device is to create a feedback loop (Figure 2): this closed-loop structure usually regulates the position or the interaction forces along a known reference (for example, a trajectory or a force field model) and can be applied either at the joint (exoskeleton) or at the end-effector level (manipulandum). This is often combined with feedforward control to compensate predictable perturbations in an anticipatory way (for example, the weight or the dynamics of the exoskeleton, and the friction forces within the joints).

Rigid position-feedback controllers combined with feedforward control are commonly used in exoskeletons (Johnson et al., 2001; Nef et al., 2007; Vertechy et al., 2009; Moubarak et al., 2010; Morales et al., 2011; Song et al., 2014). The feedforward compensation can be derived from the robot model, if available, or, since modeling the exoskeleton-human limb system is often complex, it can be learnt by using an adaptive control technique, by setting up a neural network (Yu and Rosen, 2013), or by iterative learning (Balasubramanian et al., 2008). The overall idea behind these methods is that, on the first trial no feedforward compensation is present and the feedback control must compensate for the entire error. Then, while the feedforward term is learnt trial-bytrial and increases, input from the feedback decreases, leading to better recovery from errors, in less time, with less oscillations and less rigidity on the human limb.

Feedback position controllers are sufficient for fully assistive modes of control (also known as passive mode or rigid control) or even for simple active mode control such as assistance-as-needed algorithms based on virtual guides. For example, Guidali et al. (2011) developed a virtual channel in which the subject moves: once he/she goes out of this channel, the feedback control returns $\mathrm{him} / \mathrm{her}$ to it, as if a spring was attached from the limb to the center of the virtual channel. In addition, to prevent the subject from getting stuck during the motion, a supporting force in the direction of the channel was added and adapted depending on the mean velocity of the limb. A similar idea was used by Mao and Agrawal (2012) with a cable-driven system, a low-level feedback controller and the addition of feedforward control to control the cable tension. In the study by Wolbrecht et al. (2008), the authors implemented an adaptive assisted-as-needed controller, in which a feedback controller works with a feedforward assistive term, which is adapted during the motion depending on the dynamics of the patient's limb, his/her neurological ability, and the effort he/she makes. Assistance-as-needed paradigms seem more suited for rehabilitation because the subject is pushed to make an effort to achieve the motion task. This is the key to retraining movement following stroke (Collantes et al., 2012; Pennycott et al., 2012; Krishnan et al., 2013).

A good compromise between tracking skills and the stiffness of the robot can be obtained using impedance control (Hogan, 1984). Impedance control can be seen as a force controller with position-feedback. The idea behind this controller is to regulate the relationship between tracking capacity and the rigidity of the robot by tuning the so-called mechanical impedance $Z$ :

$$
\frac{F(s)}{X(s)}=s Z(s)
$$

where $F(s)$ is the force at the interface, $X(s)$ is the output position, and $s$ refers to the Laplace transformation. The more $Z$ increases, the better $X$ is tracked, the higher $F$ is produced, and vice versa. The model of mechanical impedance $Z$ is given by the classical massspring-damper model, where the proportional gain is the spring effect, the derivative is represented by the damping factor, and the inertial mass acts as an integrator. The aim is to match this virtual model to the real interaction between the robot, the human limb, and the environment. Thus, it is a model-based method of control. This controller inverse method is admittance control, which is a position control with feedback on the force. The force-tracking trade-off feature, together with the simplicity of implementation, are the reasons why these are two of the most common control algorithms currently used for rehabilitation exoskeletons (Gupta and O'Malley, 2006; Caldwell et al., 2007; Carignan et al., 2007; Frisoli et al., 2009; Culmer et al., 2010; Yu et al., 2011). Impedance control is efficient for lightweight backdrivable exoskeletons, in which cable-driven systems are often used for torque transmission. 
Table 1 | Exoskeletons for upper-limb rehabilitation (3-DoF systems controlling at least two joints out of the shoulder-elbow-wrist chain).

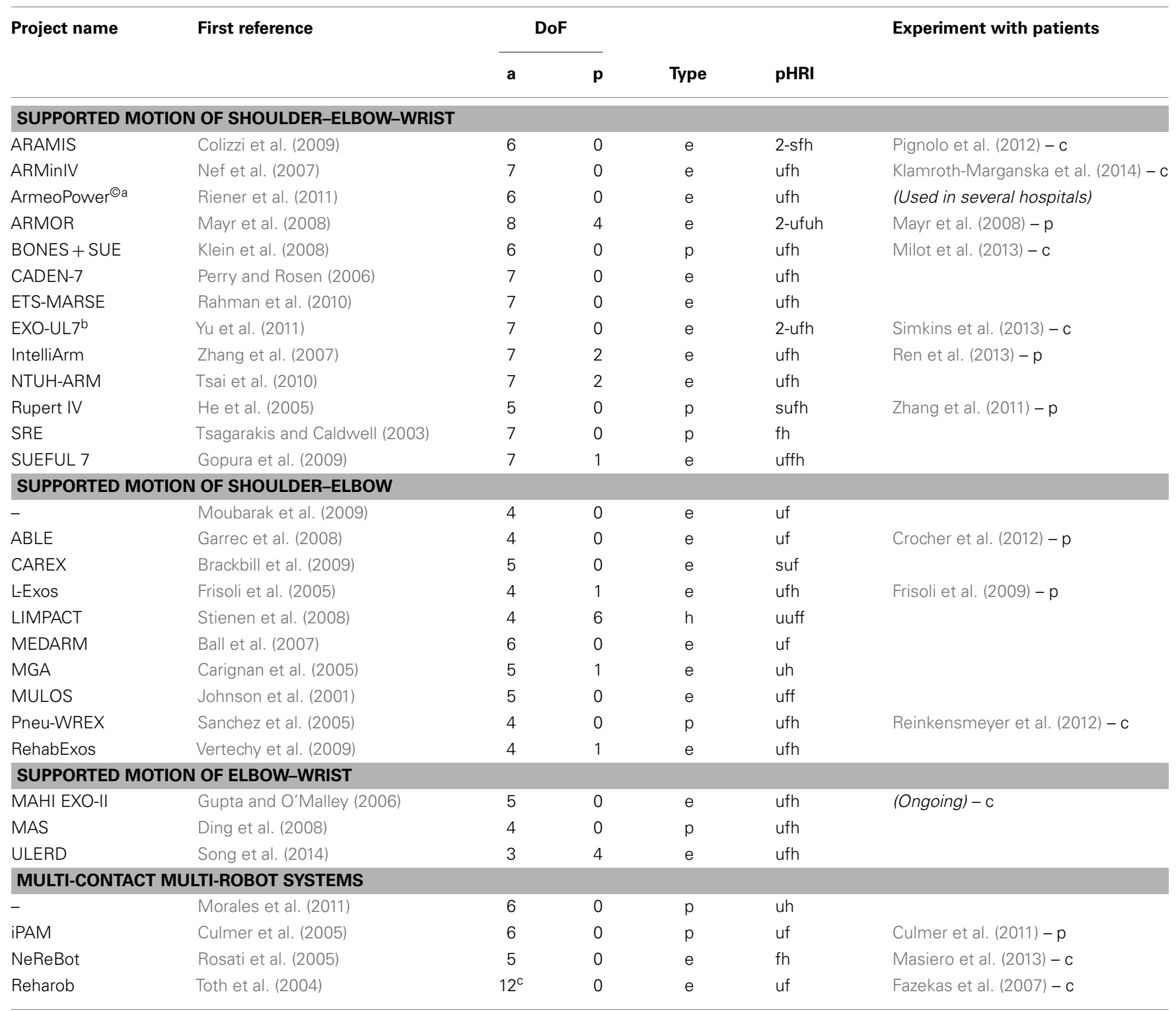

Number of degrees of freedom (DoF): a, active thus actuated; $p$, passive thus mechanical only. Type refers to the actuation system: $e$, electrical; $p$, pneumatic; $h$,

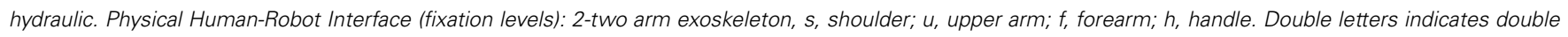
interfaces. Experiments: c, clinical; p, pre-clinical test. The last four projects are not strictly exoskeletons but rather multi-contact multi-robot systems.

a Based on ARMinll, the only commercialized exoskeleton for the clinical environment.

${ }^{b}$ Based on CADEN-7.

${ }^{\circ}$ Two 6-DoF industrial serial manipulators.

The problems relating to this type of control are the compensation of gravity and friction, particularly in tendon-like systems. For exoskeletons that lack backdrivability, admittance control may be more appropriate, because there must be measurements of the force at the interfaces with the human limb to move the robot, considering its inertia and dynamic effects.

Several research groups have attempted to use other, more complex forms of control. For example, Rahman et al. (2013) developed the Sliding Mode Control with Exponential Reaching Law (SMERL), a non-linear control mode in which the tracking problem is projected onto the state space where a sliding surface, containing the reference trajectory, is derived. The aim of the controller is to constrain the exoskeleton motion onto this surface.

Other complex approaches are based on tuning the behavior of the robot to the patient's action detected through $s E M G$ or even $E E G$ sensors. For example, Tsai et al. (2010) used an EMGbased trigger to inhibit a compensation term in an impedance controller if the electrical signal from the impaired muscles was large enough. Gopura et al. (2009) developed a neuro-fuzzy controller to determine a set of thresholds and thus a set of different 


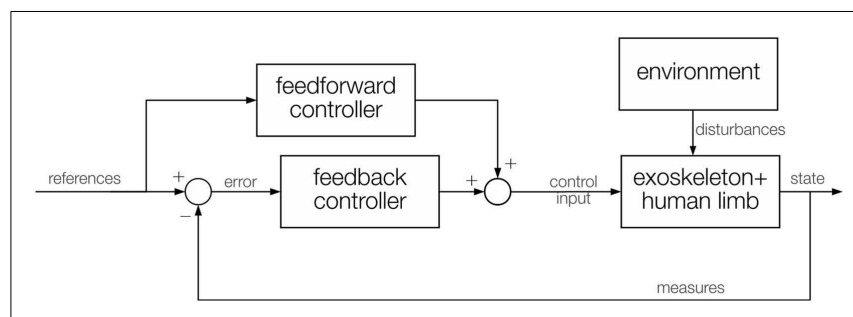

FIGURE 2 | General control scheme, with a feedback control that calculates the error and provides a control input, and a feedforward control that directly contains the desired values and provides another control input. Both inputs are combined into a single control fed to the exoskeleton actuators. Measures of the current state are fed back to the controller. The interaction with the environment acts like a disturbance on the exoskeleton control algorithm.

levels of control shared between the subject and the exoskeleton via the impedance controller, based on the subject sEMG activity. In Ding et al. (2008), after an offline estimation of muscle force using motion capture and EMG sensors, assistive torques were provided by pneumatic actuators to the impaired limb when the human joint torque was insufficient to complete the movement. In Loconsole et al. (2014), sEMG sensors were used to estimate and predict the resulting joint torques. Using this prediction, a reference position trajectory was computed and fed to a feedback controller with gravity and friction compensation. Blank et al. (2013) augmented the physical human-robot interface with a non-invasive brain-machine interface. This approach brings the human being into the control loop by capturing his/her intention of movement and anticipating the assistance required. The main drawback with all these methods is the use of sEMG/EEG to detect specific events since these sensors lack accuracy. For example, there is a poor signal to noise ratio, there can be cross-talk from other muscles, and sweat from the patient may interfere with transmission.

\subsection{CONSIDERATION OF JOINT COORDINATION IN THE EXOSKELETON CONTROL}

\subsubsection{The problem of the reference trajectory}

Most rehabilitation exoskeletons use a reference trajectory, i.e., a path and an associated velocity profile that the robot should follow. It is important to realize that the reference used by these controllers is a set of joint trajectories and not a model of joint coordination. Movements of intermediate joints either occur as a consequence of the end-effector movement in the task space, or are simply constrained along specific dedicated trajectories, which are synchronized within the joint space. These approaches thus provide simple and local solutions to the coordination problem but they do not directly address the coordination issue as a whole. Simply controlling all the joints of an exoskeleton does not address the problem of inter-joint coordination.

Defining the set of joint trajectories is also a major issue. Joint trajectories can be copied from recordings of movements in healthy subjects, informed by a therapist, or computed by an optimal trajectory planner, which, for example, relies on constraints placed on the swivel angle (around the line joining the shoulder to the wrist) to solve the redundancy of the exoskeleton and generate a suitable set of reference joint trajectories for the desired end-effector movement. However, relying on such input limits the efficacy of the control algorithms because these trajectories are generally position and time dependent and are therefore complex to generalize for different movements, targets, or tasks. This means that the patient's freedom of movement with the exoskeleton is limited as coordination patterns can only be programed for specific movements. Essentially, exoskeleton platforms are therefore reduced to simple manipulanda in $3 \mathrm{D}$ space.

\subsubsection{Coordination of joint torque}

Guidali et al. (2009) developed an approach based on correction of the pathological involuntary flexion torque, which occurs at the elbow during shoulder abduction. During an evaluation session with the robot, the patient's pathological involuntary torque was measured and a counter-active, just-as-needed torque was then calculated and applied during the therapy. A case study on one patient showed a reduction of elbow involuntary elbow flexor torque during shoulder abduction (Figure 3A) after 4 weeks of training ( $1.5 \mathrm{~h} /$ day, 3 days/week). Nevertheless, since passing from joint torque to joint kinematics is not simple and direct (because of the modulation joint stiffness modulation, among other issues), such an approach can not fully guarantee a positive and controlled effect on patient joint kinematic relationships.

Ren et al. (2013) used a similar approach with the IntelliArm exoskeleton to develop a passive stretching controller for multiple joints: individual joints are passively stretched by the robot in order to identify their individual angle-resistance torque relationships (an example of these relationships for healthy subjects and patient are shown in Figure 3B). These relationships are then used to coordinate the passive stretching of multiple joints together. Feasibility tests performed in 3 stroke patients showed a reduction of cross-coupled stiffness after a 40 min stretching session. However, no active modes of therapy (i.e., during which the patient is actively participating in the movement), based on previously identified angle-torque relationships, have been developed to target patterns of inter-joint coordination.

\subsubsection{Joint kinematic coordination}

Very few approaches have attempted to address the temporal and/or spatial relationships between joints. One approach, used with the ARMin III robot, is based on a time-independent functional training (TIFT) algorithm (Brokaw et al., 2013). This controller generates virtual joint-space walls to keep the subject close to the ideal joint-space path, acting both on multiple joint motion and feedback position control. Independence from time is important in order to allow the subject to actively achieve the task, without being constrained by rigid, planned trajectories. This strategy corrects undesirable coordination patterns between the shoulder and elbow joints. The main issue is once again the position-dependency requiring different reference paths for each joint and for each different motion. The performance of this control approach was tested on 10 moderate to severely impaired individuals with chronic stroke, and compared with results obtained with a conventional end-point tunnel algorithm (EPTT). Larger improvements in inter-joint coordination were obtained with the TIFT approach. Figure 4 shows examples of joint coordinations 

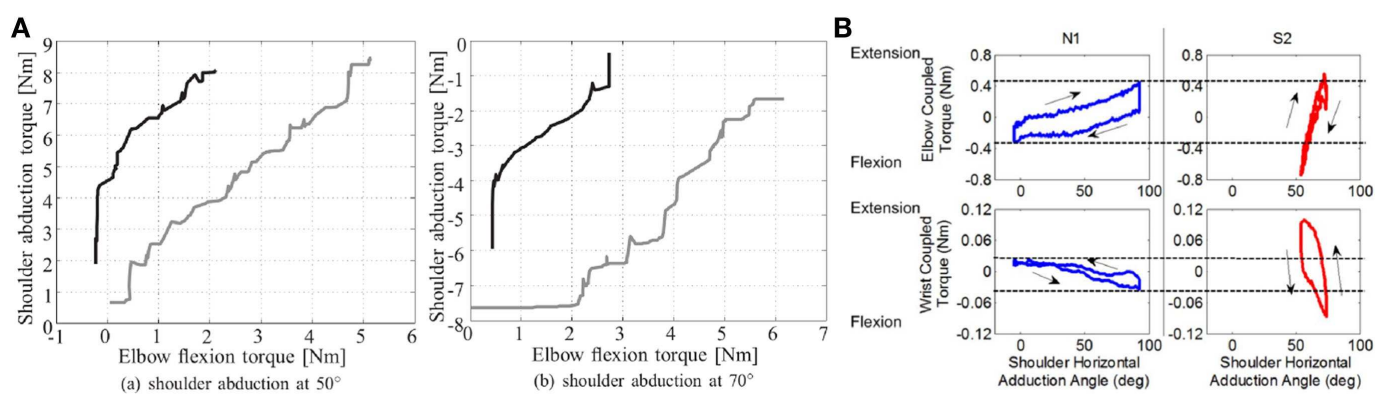

FIGURE 3 | Examples of experiments addressing joint torque coordination. (A). Decrease in the flexor synergy of one stroke patient: there was a decrease in involuntary elbow torque at $50^{\circ}$ and $70^{\circ}$ shoulder abduction at the end of the therapy. The gray lines show the torque before therapy and the black lines after (Guidali et al., 2011). (B). Measures of elbow and wrist coupled torques as a function of the shoulder abduction angle for a healthy subject (N1) and a stroke survivor (S2) (Ren et al., 2013).
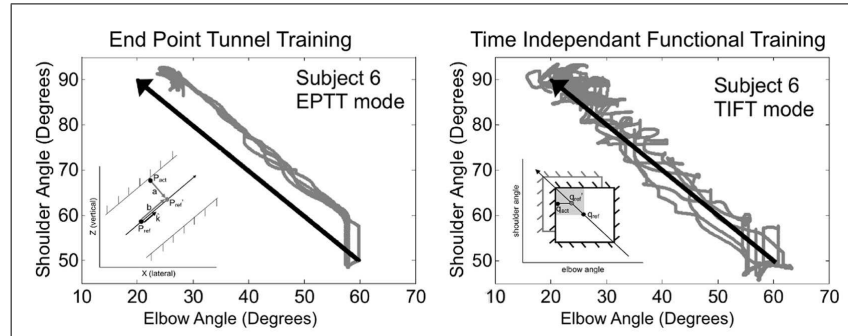

FIGURE 4 | Comparison of typical training trajectories from one stroke patient obtained with conventional end-point tunnel control (EPTT) and time independent functional training (TIFT) (Brokaw et al., 2013). In TIFT, subjects' trajectories tend to be close to the ideal desired path. Illustrations of the principle of the movement constraints are shown within each figure (EPTT imposes constraints at the cartesien end-effector level while TIFT imposes them at the joint level).

obtained for one subject with both control approaches, along with a representation of associated control approaches.

An alternative approach has been presented in Crocher et al. (2012). In this work, the problem of tracking is projected from the reference trajectory space to the joint velocities synergy space. The exoskeleton generates reactive viscous joint torques to impose specific patterns of inter-joint coordination without constraining hand motion.

For this approach, a first step consists of characterizing the inter-joint coordination required to achieve a given task. This characterization is performed using principal component analysis (PCA) in the joint velocity space. This technique is widely used in the study of synergies. PCA provides a different approach to the expression of joint velocity space by summarizing the $n_{r}$ (the number of considered joints) dimensions through principal component vectors $\mathbf{p}_{1}$ to $\mathbf{p}_{\mathbf{n}_{\mathbf{r}}}$. Since the human arm has a redundant number of DoF for the performance of all common tasks (e.g., a reaching task in $3 \mathrm{D}$ requires only $3-\mathrm{DoF}$ since the orientation is not constrained), only $m<n_{r}$ first principal components are required to fully represent the task to perform. The remaining $n_{r}-m$ components thus represent the unnecessary coordinations for the given task. The pathological synergies, or abnormal coordination, thus, "lie within" these latest components: $\left[\mathbf{p}_{\mathbf{m}+\mathbf{1}} \cdots \mathbf{p}_{\mathbf{n}_{\mathbf{r}}}\right]$, which are used to define a movement constraint matrix $\mathbf{C} \in \mathbb{R}^{\left(n_{r}-m\right) \times n_{r}}$ :

$$
\mathbf{C}=\left[\begin{array}{c}
\mathbf{p}_{\mathbf{m}+\mathbf{1}} \\
\vdots \\
\mathbf{p}_{\mathbf{n}_{\mathbf{r}}}
\end{array}\right]=\left[\begin{array}{c}
\mathbf{p}_{4} \\
\vdots \\
\mathbf{p}_{\mathbf{n}_{\mathbf{r}}}
\end{array}\right] \text { (for a } 3 \text { DoF task) }
$$

A robotics control method is then used to constrain the movement outwith this unnecessary subspace such that at any given time instant:

$$
\mathbf{C} \dot{\mathbf{q}}_{\mathbf{r}}=0
$$

where $\dot{\mathbf{q}}_{\mathrm{r}} \in \mathbb{R}^{n_{r}}$ is the joint velocity vector. The subject should thus produce a well coordinated movement with a velocity vector respecting the given constraint $\mathbf{C}$. A correction is calculated for any velocity vector outside of the subspace necessary to achieve the task with the defined coordination. The corrective torque is a viscous field bringing the movement back into this desired movement subspace:

$$
\tau_{\mathbf{c}}=-b \mathbf{C}^{+} \mathbf{C} \dot{\mathbf{q}}_{\mathbf{r}}
$$

where $b$ is a scalar viscosity coefficient tuning the intensity of the correction. The exoskeleton torques $\tau_{c}$ will be null when the subject's movement respects the defined coordination and will correct the movement without explicitly affecting the hand movement otherwise.

This approach was first experimented on a 4 DoF upperlimb exoskeleton (ABLE exoskeleton - a transparent screw-cable mechanical transmission based device, with 3-DoF for the shoulder and 1 for the elbow developed by CEA-LIST (Garrec et al., 2008) with healthy subjects performing 3-dimensional reaching tasks and showed its ability to impose un-natural synergies on healthy subjects without altering hand motion (Crocher et al., 2011).

Preliminary experiments were also conducted with hemiparetic patients: before carrying out the task, a therapist passively guided the patient's arm (wearing the robot in transparent mode) toward the targets, ensuring that the joints followed a normal pattern of 


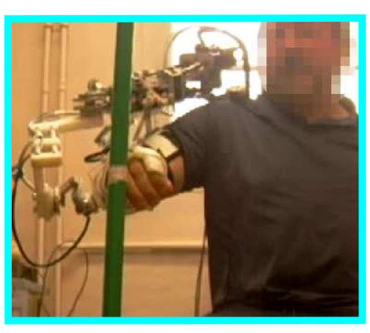

Transparent robot

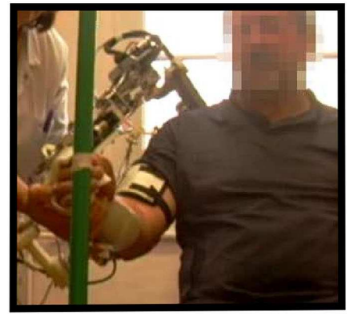

Therapist (patient inactive)

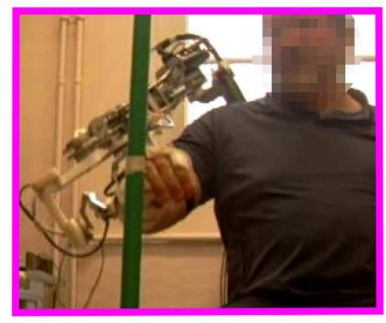

Therapeutic constraint

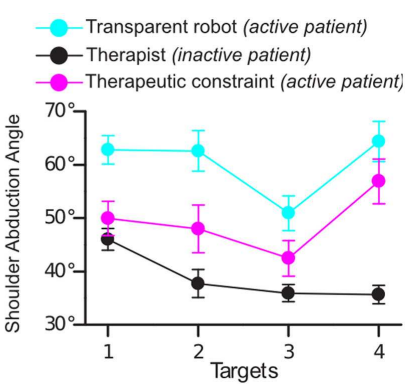

transparent mode) and with robot applying the therapeutic constraint (Crocher et al., 2012). Right: final abduction angle of robot + arm for each target (external, middle, internal, and high) averaged for all patients, measured in the different modes. coordination. This pattern of coordination was then used to define the matrix $\mathrm{C}$ of the active-constrained synergy-based controller. The results showed that the controller could impose constraints in the same way a therapist would do. In other words, the exoskeleton shaped the patient's movement similarly to the therapist. As can be seen in Figure 5, the therapeutic constraint generated by the controller decreased shoulder abduction (part of an abnormal synergy), without significantly modifying the endpoint trajectory. This improvement in the upper-limb synergy while preserving the patient's movement intention is a common rehabilitation goal. These preliminary studies, however, did not involve enough movement repetition or measures of post-effects to evaluate any learning effects or to make conclusions regarding the effect of this novel form of rehabilitation compared to conventional therapy.

\section{DISCUSSION AND PERSPECTIVES}

Therapy with exoskeletons can theoretically combine motor learning principles, which insist on the importance of intensive therapy using active movements, and the more classical methods, which are based on improving the quality of coordination. Some simple technological rehabilitation devices have recently been developed for this purpose. Most focus on one joint or a local group of joints such as hand or wrist rehabilitation robots or simple hand motion analysis systems (like inertial measurement units (IMU) or the Nintendo $\mathrm{Wii}^{\odot}$ ). These devices are, however, rarely functional and may lead the patient to develop compensatory strategies.

The use of a multi-contact robotic device for the rehabilitation of coordination thus seems promising. However, as explained in this paper, the development of exoskeletons for rehabilitation is only beginning and numerous technological, physiological, and clinical challenges lie ahead. With regard to the results of clinical investigations, the few studies, which have directly addressed the temporal and spatial relationships between joints have only been preliminary, involving a limited number of patients (listed in Section 3.3). Only the ARMin IV exoskeleton (KlamrothMarganska et al., 2014) has so far been tested in a randomized controlled clinical trial. However, this study did not directly address coordination since the control used was the classical "assist-asneeded" method, along a pre-defined set of joint trajectories. The patients underwent $45 \mathrm{~min}$ of robot therapy, 3 times a week for 8 weeks during the chronic stage of stroke ( $>6$ months) and the results showed a statistically significant improvement of the Fugl-Meyer score, however, the improvement was not clinically significant. In addition the benefit was not maintained at followup. The strength was not modified by therapy. This result is similar to a clinical trial involving the TWrex system (commercial name: Armeo Spring ${ }^{\odot}$ ), which is not motorized but uses a system of springs to assist arm movement (Housman et al., 2009). The relatively disappointing results of these two studies might be due to the chronic stage of the hemiparesis and/or to the limited duration of the therapy.

The current lack of positive results is not surprising, for several reasons. First, only a few devices currently have the high level of transparency, which is essential for the rehabilitation of inter-joint coordination with smooth corrective mechanisms similar to clinical practice. During Bobath therapy, the patient is active, occasionally guided or corrected, but not constantly constrained to follow a fixed gesture. In contrast, poorly transparent robots impose (more or less rigidly) constant constraints with pre-defined coordination or tunneling. This mode of interaction is less physiological and its efficiency is questionable.

The second reason is the lack of simple tools and metrics to assess inter-joint coordination. The clinical scales commonly used may not have been sensitive enough to evaluate specific improvements. More precise quantitative methods may be more adapted to evaluate changes and follow-up (Jarrasse et al., 2010; Kim et al., 2013), to specify indications for such therapy and later to determine the optimal dose and duration. Finally, the control of the redundant, multi-joint limbs by the CNS is still poorly understood (Guigon et al., 2007; Martin et al., 2009). It is therefore more difficult to incorporate an automatized generator of joint coordination trajectories in exoskeletons than it is to implement "human-like" end-effector trajectories (based, for example, on the minimum jerk theory) in manipulanda, since no consensual and simple model of redundancy resolution is available.

Indeed, the number of studies on the effect of perturbative or assistive fields at the inter-joint level is limited. Most experiments on physical interaction have been performed using planar manipulanda with 2D force fields and a single point of attachment between the subject and the robot. Translation of knowledge and methods from the research to the clinical environment was therefore simple for manipulanda, as both used similar platforms. Exoskeletons are 
still little used for research in motor control, despite the fact that it is critical to generalize existing motor control theories to a more complex framework including 3D force fields acting at the joint level with multiple interaction points. Studies of the after-effects, which occur following the application of joint constraints with an exoskeleton has, for example, never been studied in healthy subjects. Advances in these scientific fields should have an important impact on clinical exoskeletons, leading to the design of innovative approaches to rehabilitation with the training of inter-joint coordination. This improvement at the impairment level should limit the development of compensatory strategies, helping patients to reach their full functional potential (Taub et al., 2006; Levin et al., 2009).

\section{AUTHOR CONTRIBUTIONS}

Nathanael Jarrassé, Tommaso Proietti, Vincent Crocher, and Agnes Roby-Brami wrote the paper. Nathanael Jarrassé and Guillaume Morel authored the work presented in Section 2.2. Vincent Crocher, Johanna Robertson, Anis Sahbani, Agnes Roby-Brami, and Guillaume Morel authored the work presented in Section 3.4. Johanna Robertson and Agnes Roby-Brami carried out the related clinical experiments. All authors read and approved the submitted manuscript.

\section{ACKNOWLEDGMENTS}

The authors thank Catherine Bidard, Philippe Garrec, and Yann Perrot (CEA-LIST) for their contribution with the ABLE Exoskeleton and the participants of the European Network on Robotics for NeuroRehabilitation (COST action TD1006) for fruitful discussions. We also thank Prof. Djamel Bensmail and Prof. Bernard Bussel (Hôpital Raymond Poincaré Garches) for their support with clinical experiments. Funding: This work was supported by the French National Agency for research, ANR, program PSIROBROBO-0003. Tommaso Proietti received a doctoral grant from Region Ile de France.

\section{REFERENCES}

Balasubramanian, S., Wei, R., and He, J. (2008). Rupert closed loop control design. Conf. Proc. IEEE Eng. Med. Biol. Soc. 2008, 3467-3470. doi:10.1109/IEMBS.2008. 4649952

Ball, S., Brown, I., and Scott, S. (2007). Medarm: A Rehabilitation Robot with 5 dof at the Shoulder Complex. Zurich: IEEE, 1-6.

Beer, R. F., Dewald, J. P., Dawson, M. L., and Rymer, W. Z. (2004). Target-dependent differences between free and constrained arm movements in chronic hemiparesis. Exp. Brain Res. 156, 458-470. doi:10.1007/s00221-003-1807-8

Beer, R. F., Ellis, M. D., Holubar, B. G., and Dewald, J. P. (2007). Impact of gravity loading on post-stroke reaching and its relationship to weakness. Muscle Nerve 36, 242-250. doi:10.1002/mus.20817

Bernstein, N. (1967). The Coordination and Regulation of Movement. London: Pergamon Press.

Bizzi, E., Cheung, V. C., d'Avella, A., Saltiel, P., and Tresch, M. (2008). Combining modules for movement. Brain Res. Rev. 57, 125-133. doi:10.1016/j.brainresrev. 2007.08.004

Blank, A., O’Malley, M. K., Francisco, G. E., and Contreras-Vidal, J. L. (2013). A PreClinical Framework for Neural Control of a Therapeutic Upper-Limb Exoskeleton. San Diego, CA: IEEE, 1159-1162.

Bobath, B. (1990). Adult Hemiplegia: Evaluation and Treatment. London: Butterworth-Heinemann Medical.

Brackbill, E. A., Mao, Y., Agrawal, S. K., Annapragada, M., and Dubey, V. N. (2009). Dynamics and Control of a 4-dof Wearable Cable-Driven Upper Arm Exoskeleton. Kobe: IEEE, 2300-2305.
Brewer, B. R., McDowell, S. K., and Worthen-Chaudhari, L. C. (2007). Poststroke upper extremity rehabilitation: a review of robotic systems and clinical results. Top. Stroke Rehabil. 14, 22-44. doi:10.1310/tsr1406-22

Brokaw, E. B., Holley, R. J., and Lum, P. S. (2013). Comparison of joint space and end point space robotic training modalities for rehabilitation of interjoint coordination in individuals with moderate to severe impairment from chronic stroke. IEEE Trans. Neural Syst. Rehabil. Eng. 21, 787-795. doi:10.1109/TNSRE.2013. 2238251

Brunnstrom, S. (1970). Movement Therapy in Hemiplegia: A Neurophysiological Approach. New York, NY: Harper \& Row.

Caldwell, D. G., Tsagarakis, N. G., Kousidou, S., Costa, N., and Sarakoglou, I. (2007). Soft exoskeleton for upper and lower body rehabilitation - desing, control and testing. Int. J. HR. 4, 549-573. doi:10.1142/S0219843607001151

Capaday, C. (2004). The integrated nature of motor cortical function. Neuroscientist. 10, 207-220. doi:10.1177/107385403262109

Carignan, C., Liszka, M., and Roderick, S. (2005). Design of an Arm Exoskeleton with Scapula Motion for Shoulder Rehabilitation. Seattle, WA: IEEE, 524-531.

Carignan, C., Tang, J., Roderick, S., and Naylor, M. (2007). A Configuration-Space Approach to Controlling a Rehabilitation Arm Exoskeleton. Noordwijk: IEEE, 179-187.

Cheney, P. D., and Fetz, E. E. (1985). Comparable patterns of muscle facilitation evoked by individual corticomotoneuronal (CM) cells and by single intracortical microstimuli in primates: evidence for functional groups of $\mathrm{cm}$ cells. $J$. Neurophysiol. 53, 786-804.

Cheung, V. C., Piron, L., Agostini, M., Silvoni, S., Turolla, A., and Bizzi, E. (2009). Stability of muscle synergies for voluntary actions after cortical stroke in humans. Proc. Natl. Acad. Sci. U.S.A. 106, 19563-19568. doi:10.1073/pnas. 0910114106

Cheung, V. C., Turolla, A., Agostini, M., Silvoni, S., Bennis, C., Kasi, P., et al. (2012). Muscle synergy patterns as physiological markers of motor cortical damage. Proc. Natl. Acad. Sci. U.S.A. 109, 14652-14656. doi:10.1073/pnas.1212056109

Cirstea, M. C., and Levin, M. F. (2000). Compensatory strategies for reaching in stroke. Brain 123, 940-953. doi:10.1093/brain/123.5.940

Cirstea, M. C., Mitnitski, A. B., Feldman, A. G., and Levin, M. F. (2003). Interjoint coordination dynamics during reaching in stroke. Exp. Brain Res. 151, 289-300. doi:10.1007/s00221-003-1438-0

Colebatch, J. G., and Gandevia, S. C. (1989). The distribution of muscular weakness in upper motor neuron lesions affecting the arm. Brain 112(Pt 3), 749-763. doi:10.1093/brain/112.3.749

Colizzi, L., Lidonnici, A., and Pignolo, L. (2009). The aramis project: a concept robot and technical design. J. Rehabil. Med. 41, 1011-1101. doi:10.2340/165019770407

Collantes, I., Asín, G., Moreno, J., and Pons, J. (2012). "Analysis of biomechanical data to determine the degree of users participation during robotic-assisted gait rehabilitation," in Engineering in Medicine and Biology Society (EMBC), 2012 Annual International Conference of the IEEE (San Diego: IEEE), 4855-4858.

Crocher, V., Jarrasse, N., Sahbani, A., Roby-Brami, A., and Morel, G. (2011). Changing Human Upper-Limb Synergies with an Exoskeleton Using Viscous Fields. Shanghai: IEEE, 4657-4663.

Crocher, V., Sahbani, A., Robertson, J., Roby-Brami, A., and Morel, G. (2012). Constraining upper limb synergies of hemiparetic patients using a robotic exoskeleton in the perspective of neuro-rehabilitation. IEEE Trans. Neural Syst. Rehabil. Eng. 20, 247-257. doi:10.1109/TNSRE.2012.2190522

Culmer, P., Jackson, A., Makower, S., Richardson, R., Cozens, J., Levesley, M., et al. (2010). A control strategy for upper limb robotic rehabilitation with a dual robot system. IEEE/ASME Trans. Mechatron. 15, 575-585. doi:10.1109/TMECH.2009. 2030796

Culmer, P., Jackson, A., Richardson, R., Bhakta, B., Levesley, M., and Cozens, A. (2005). Development of a Dual Robotic System for Upper-Limb Stroke Rehabilitation. IEEE 61-65.

Culmer, P. R., Jackson, A. E., Makower, S. G., Cozens, J. A., Levesley, M. C., MonWilliams, M., et al. (2011). A novel robotic system for quantifying arm kinematics and kinetics: description and evaluation in therapist-assisted passive arm movements post-stroke. J. Neurosci. Methods 197, 259-269. doi:10.1016/j.jneumeth. 2011.03.004

d'Avella, A., Fernandez, L., Portone, A., and Lacquaniti, F. (2008). Modulation of phasic and tonic muscle synergies with reaching direction and speed. J. Neurophysiol. 100, 1433-1454. doi:10.1152/jn.01377.2007 
d'Avella, A., Portone, A., Fernandez, L., and Lacquaniti, F. (2006). Control of fast-reaching movements by muscle synergy combinations. J. Neurosci. 26, 7791-7810. doi:10.1523/JNEUROSCI.0830-06.2006

Dewald, J. P., and Beer, R. F. (2001). Abnormal joint torque patterns in the paretic upper limb of subjects with hemiparesis. Muscle Nerve 24, 273-283. doi:10.1002/1097-4598(200102)24:2<273::AID-MUS130>3.0.CO;2-Z

Dewald, J. P., Beer, R. F., Given, J. D., McGuire, J. R., and Rymer, W. Z. (1999). Reorganization of flexion reflexes in the upper extremity of hemiparetic subjects. Muscle Nerve 22, 1209-1221. doi:10.1002/(SICI)1097-4598(199909)22:9<1209: :AID-MUS7>3.0.CO;2-B

Dewald, J. P., Pope, P. S., Given, J. D., Buchanan, T. S., and Rymer, W. Z. (1995). Abnormal muscle coactivation patterns during isometric torque generation at the elbow and shoulder in hemiparetic subjects. Brain 118(Pt 2), 495-510. doi:10.1093/brain/118.2.495

Ding, M., Ueda, J., and Ogasawara, T. (2008). "Pinpointed muscle force control using a power-assisting device: system configuration and experiment," in 2nd IEEE RAS \& EMBS International Conference on Biomedical Robotics and Biomechatronics, 2008. BioRob 2008 (IEEE), 181-186.

Dipietro, L., Krebs, H. I., Fasoli, S. E., Volpe, B. T., Stein, J., Bever, C., et al. (2007). Changing motor synergies in chronic stroke. J. Neurophysiol. 98, 757-768. doi:10.1152/jn.01295.2006

Ellis, M. D., Drogos, J., Carmona, C., Keller, T., and Dewald, J. P. (2012). Neck rotation modulates flexion synergy torques, indicating an ipsilateral reticulospinal source for impairment in stroke. J. Neurophysiol. 108, 3096-3104. doi:10.1152/jn.01030.2011

Ellis, M. D., Sukal, T., DeMott, T., and Dewald, J. P. (2008). Augmenting clinical evaluation of hemiparetic arm movement with a laboratory-based quantitative measurement of kinematics as a function of limb loading. Neurorehabil. Neural Repair 22, 321-329. doi:10.1177/1545968307313509

Ellis, M. D., Sukal-Moulton, T., and Dewald, J. P. (2009). Progressive shoulder abduction loading is a crucial element of arm rehabilitation in chronic stroke. $\mathrm{Neu}$ rorehabil. Neural Repair 23, 862-869. doi:10.1177/1545968309332927

Fazekas, G., Horvath, M., Troznai, T., and Toth, A. (2007). Robot-mediated upper limb physiotherapy for patients with spastic hemiparesis: a preliminary study. $J$. Rehabil. Med. 39, 580-582. doi:10.2340/16501977-0087

Feldman, A. G., Goussev, V., Sangole, A., and Levin, M. F. (2007). Threshold position control and the principle of minimal interaction in motor actions. Prog. Brain Res. 165, 267-281. doi:10.1016/S0079-6123(06)65017-6

Frisoli, A., Procopio, C., Chisari, C., Creatini, I., Bonfiglio, L., Bergamasco, M., et al. (2012). Positive effects of robotic exoskeleton training of upper limb reaching movements after stroke. J. Neuroeng. Rehabil. 9, 36. doi:10.1186/1743-00039-36

Frisoli, A., Rocchi, F., Marcheschi, S., Dettori, A., Salsedo, F., and Bergamasco, M. (2005). "A new force-feedback arm exoskeleton for haptic interaction in virtual environments," in Eurohaptics Conference, 2005 and Symposium on Haptic Interfaces for Virtual Environment and Teleoperator Systems, 2005 (IEEE), 195-201.

Frisoli, A., Salsedo, F., Bergamasco, M., Rossi, B., and Carboncini, M. C. (2009). A force-feedback exoskeleton for upper-limb rehabilitation in virtual reality. Appl. Bionics Biomech. 6, 115-126. doi:10.1080/11762320902959250

Galinski, D., Sapin, J., and Dehez, B. (2013). "Optimal design of an alignment-free two-dof rehabilitation robot for the shoulder complex," in 2013 IEEE International Conference on Rehabilitation Robotics (ICORR) (IEEE), 1-7.

Garrec, P., Friconneau, J., Measson, Y., and Perrot, Y. (2008). "Able, an innovative transparent exoskeleton for the upper-limb," in IROS 2008. IEEE/RSJ International Conference on Intelligent Robots and Systems, 2008 (IEEE), 1483-1488.

Gomi, H., and Kawato, M. (1997). Human arm stiffness and equilibrium-point trajectory during multi-joint movement. Biol. Cybern. 76, 163-171. doi:10.1007/ s004220050329

Gopura, R. A. R. C., Kiguchi, K., and Li, Y. (2009). "Sueful-7: a 7dof upper-limb exoskeleton robot with muscle-model-oriented emg-based control," in IEEE/RSJ International Conference on Intelligent Robots and Systems, 2009, IROS 2009 (IEEE), 1126-1131.

Gracies, J. M. (2005). Pathophysiology of spastic paresis. ii: emergence of muscle overactivity. Muscle Nerve 31, 552-571. doi:10.1002/mus.20285

Guidali, M., Duschau-Wicke, A., Broggi, S., Klamroth-Marganska, V., Nef, T., and Riener, R. (2011). A robotic system to train activities of daily living in a virtual environment. Med. Biol. Eng. Comput. 49, 1213-1223. doi:10.1007/s11517-0110809-0
Guidali, M., Schmiedeskamp, M., Klamroth, V., and Riener, R. (2009). “Assessment and training of synergies with an arm rehabilitation robot," in IEEE International Conference on, Rehabilitation Robotics, 2009. ICORR 2009 (IEEE), 772-776.

Guigon, E., Baraduc, P., and Desmurget, M. (2007). Computational motor control: redundancy and invariance. J. Neurophysiol. 97, 331-347. doi:10.1152/jn.00290. 2006

Gupta, A., and O'Malley, M. (2006). Design of a haptic arm exoskeleton for training and rehabilitation. IEEE/ASME Trans. Mechatron. 11, 280-289. doi:10.1109/ TMECH.2006.875558

He, J., Koeneman, E., Schultz, R., Herring, D., Wanberg, J., Huang, H., et al. (2005). "Rupert: a device for robotic upper extremity repetitive therapy," in 27th Annual International Conference of the IEEE Engineering in Medicine and Biology Society, 2005, IEEE-EMBS 2005 (IEEE), 6844-6847.

Hidaka, Y., Han, C. E., Wolf, S. L., Winstein, C. J., and Schweighofer, N. (2012). Use it and improve it or lose it: interactions between arm function and use in humans post-stroke. PLoS Comput. Biol. 8:e1002343. doi:10.1371/journal.pcbi.1002343

Hogan, N. (1984). "Impedance control: an approach to manipulation," in American Control Conference, 1984 (IEEE), 304-313. doi:10.1115/1.3140702

Hogan, N., and Krebs, H. I. (2004). Interactive robots for neuro-rehabilitation. Restor. Neurol. Neurosci. 22, 349-358.

Hogan, N., Krebs, H. I., Rohrer, B., Palazzolo, J. J., Dipietro, L., Fasoli, S. E., et al. (2006). Motions or muscles? Some behavioral factors underlying robotic assistance of motor recovery. J. Rehabil. Res. Dev. 43, 605-618. doi:10.1682/JRRD. 2005.06.0103

Honeycutt, C. F., and Perreault, E. J. (2012). Planning of ballistic movement following stroke: insights from the startle reflex. PLoS ONE 7:e43097. doi:10.1371/ journal.pone.0043097

Housman, S., Scott, K., and Reinkensmeyer, D. (2009). A randomized controlled trial of gravity-supported, computer-enhanced arm exercise for individuals with severe hemiparesis. Neurorehabil. Neural Repair. 23, 505-514. doi:10.1177/ 1545968308331148

Housman, S., Vu, L., Rahman, T., Sanchez, R., and Reinkensmeyer, D. (2007). "Armtraining with T-WREX after chronic stroke: preliminary results of a randomized controlled trial," in IEEE 10th International Conference on Rehabilitation Robotics, 2007. ICORR 2007 (IEEE), 562-568.

Huang, V., and Krakauer, J. (2009). Robotic neurorehabilitation: a computational motor learning perspective. J. Neuroeng. Rehabil. 6, 5. doi:10.1186/1743-00036-5

Jarrassé, N., and Morel, G. (2012). Connecting a Human Limb to An Exoskeleton, Vol. 28. IEEE, 697-709.

Jarrasse, N., Tagliabue, M., Robertson, J., Maiza, A., Crocher, V., Roby-Brami, A., et al. (2010). A methodology to quantify alterations in human upper limb movement during co-manipulation with an exoskeleton. IEEE Trans. Neural Syst. Rehabil. Eng. 18, 389-397. doi:10.1109/TNSRE.2010.2056388

Johnson, G. R., Carus, D. A., Parrini, G., Marchese, S., and Valeggi, R. (2001). The design of a five-degree-of-freedom powered orthosis for the upper limb. Proc. Inst. Mech. Eng. H 215, 275-284. doi:10.1243/0954411011535867

Kim, H., Miller, L. M., Fedulow, I., Simkins, M., Abrams, G. M., Byl, N., et al. (2013). Kinematic data analysis for post-stroke patients following bilateral versus unilateral rehabilitation with an upper limb wearable robotic system. IEEE Trans. Neural Syst. Rehabil. Eng. 21, 153-164. doi:10.1109/TNSRE.2012.2207462

Kitago, T., Liang, J., Huang, V. S., Hayes, S., Simon, P., Tenteromano, L., et al. (2013). Improvement after constraint-induced movement therapy: recovery of normal motor control or task-specific compensation? Neurorehabil. Neural Repair 27, 99-109. doi:10.1177/1545968312452631

Klamroth-Marganska, V., Blanco, J., Campen, K., Curt, A., Dietz, V., Ettlin, T., et al. (2014). Three-dimensional, task-specific robot therapy of the arm after stroke: a multicentre, parallel-group randomised trial. Lancet Neurol 13, 159-166. doi:10.1016/S1474-4422(13)70305-3

Klein, J., Spencer, S., Allington, J., Minakata, K., Wolbrecht, E., Smith, R., et al. (2008). "Biomimetic orthosis for the neurorehabilitation of the Elbow and Shoulder (Bones)," in 2nd IEEE RAS \& EMBS International Conference on Biomedical Robotics and Biomechatronics, 2008, BioRob 2008 (IEEE), 535-541.

Krabben, T., Prange, G. B., Molier, B. I., Stienen, A. H., Jannink, M. J., Buurke, J. H., et al. (2012). Influence of gravity compensation training on synergistic movement patterns of the upper extremity after stroke, a pilot study. J. Neuroeng. Rehabil. 9, 44. doi:10.1186/1743-0003-9-44

Krishnan, C., Kotsapouikis, D., Dhaher, Y. Y., and Rymer, W. Z. (2013). Reducing robotic guidance during robot-assisted gait training improves gait function 
a case report on a stroke survivor. Arch. Phys. Med. Rehabil. 94, 1202-1206. doi:10.1016/j.apmr.2012.11.016

Lance, J. W. (1980). The control of muscle tone, reflexes, and movement: Robert Wartenberg lecture. Neurology 30, 1303-1313. doi:10.1212/WNL.30.12.1303

Langhorne, P., Bernhardt, J., and Kwakkel, G. (2011). Stroke rehabilitation. Lancet 377, 1693-1702. doi:10.1016/S0140-6736(11)60325-5

Latash, M. L., and Anson, J. G. (2006). Synergies in health and disease: relations to adaptive changes in motor coordination. Phys. Ther. 86, 1151-1160.

Levin, M. F. (1996). Interjoint coordination during pointing movements is disrupted in spastic hemiparesis. Brain 119(Pt 1), 281-293. doi:10.1093/brain/119. 1.281

Levin, M. F., and Dimov, M. (1997). Spatial zones for muscle coactivation and the control of postural stability. Brain Res. 757, 43-59. doi:10.1016/S0006-8993(97) 00204-7

Levin, M. F., Kleim, J. A., and Wolf, S. L. (2009). What do motor "recovery" and "compensation" mean in patients following stroke? Neurorehabil. Neural Repair 23, 313-319. doi:10.1177/1545968308328727

Levin, M. F., and Panturin, E. (2011). Sensorimotor integration for functional recovery and the Bobath approach. Motor Control 15, 285-301.

Lo, A. C., Guarino, P. D., Richards, L. G., Haselkorn, J. K., Wittenberg, G. F., Federman, D. G., et al. (2010). Robot-assisted therapy for long-term upperlimb impairment after stroke. N. Engl. J. Med. 362, 1772-1783. doi:10.1056/ NEJMoa0911341

Lo, H. S., and Xie, S. Q. (2012). Exoskeleton robots for upper-limb rehabilitation: state of the art and future prospects. Med. Eng. Phys. 34, 261-268. doi:10.1016/j.medengphy.2011.10.004

Loconsole, C., Dettori, S., Frisoli, A., Avizzano, C. A., and Bergamasco, M. (2014). "An EMG-based approach for on-line predicted torque control in robotic-assisted rehabilitation," in Haptics Symposium (HAPTICS), 2014 (IEEE), $181-186$.

Lynch, D., Ferraro, M., Krol, J., Trudell, C. M., Christos, P., and Volpe, B. T. (2005). Continuous passive motion improves shoulder joint integrity following stroke. Clin. Rehabil. 19, 594-599. doi:10.1191/0269215505cr901oa

Maciejasz, P., Eschweiler, J., Gerlach-Hahn, K., Jansen-Troy, A., and Leonhardt, S. (2014). A survey on robotic devices for upper limb rehabilitation. J. Neuroeng. Rehabil. 11, 3. doi:10.1186/1743-0003-11-3

Maclean, N., Pound, P., Wolfe, C., and Rudd, A. (2000). Qualitative analysis of stroke patients' motivation for rehabilitation. BMJ 321, 1051-1054. doi:10.1136/bmj. 321.7268 .1051

Mao, Y., and Agrawal, S. (2012). Design of a cable-driven arm exoskeleton (carex) for neural rehabilitation. IEEE Trans. Robot. 28, 922-931. doi:10.1109/TRO.2012. 2189496

Marchal-Crespo, L., and Reinkensmeyer, D. J. (2009). Review of control strategies for robotic movement training after neurologic injury. J. Neuroeng. Rehabil. 6, 20. doi:10.1186/1743-0003-6-20

Martin, V., Scholz, J. P., and Schoner, G. (2009). Redundancy, self-motion, and motor control. Neural Comput. 21, 1371-1414. doi:10.1162/neco.2008.0108-698

Masiero, S., Armani, M., Ferlini, G., Rosati, G., and Rossi, A. (2013). Randomized trial of a robotic assistive device for the upper extremity during early inpatient stroke rehabilitation. Neurorehabil. Neural Repair. 28, 377-386. doi:10.1177/1545968313513073

Mayr, A., Kofler, M., and Saltuari, L. (2008). Armor: elektromechanischer roboter für das bewegungstraining der oberen extremität nach schlaganfall. prospektive randomisierte kontrollierte pilotstudie. Handchirurgie Mikrochirurgie Plastische Chirurgie 40, 66-73. doi:10.1055/s-2007-989425

Mazevet, D., Meunier, S., Pradat-Diehl, P., Marchand-Pauvert, V., and PierrotDeseilligny, E. (2003). Changes in propriospinally mediated excitation of upper limb motoneurons in stroke patients. Brain 126, 988-1000. doi:10.1093/brain/ awg088

McCrea, P. H., Eng, J. J., and Hodgson, A. J. (2005). Saturated muscle activation contributes to compensatory reaching strategies after stroke. J. Neurophysiol. 94, 2999-3008. doi:10.1152/jn.00732.2004

Merdler, T., Liebermann, D. G., Levin, M. F., and Berman, S. (2013). Armplane representation of shoulder compensation during pointing movements in patients with stroke. J. Electromyogr. Kinesiol. 23, 938-947. doi:10.1016/j.jelekin. 2013.03.006

Michaelsen, S. M., Dannenbaum, R., and Levin, M. F. (2006). Task-specific training with trunk restraint on arm recovery in stroke: randomized control trial. Stroke 37, 186-192. doi:10.1161/01.STR.0000196940.20446.c9
Michaelsen, S. M., Luta, A., Roby-Brami, A., and Levin, M. F. (2001). Effect of trunk restraint on the recovery of reaching movements in hemiparetic patients. Stroke 32, 1875-1883. doi:10.1161/01.STR.32.8.1875

Milot, M.-H., Spencer, S. J., Chan, V., Allington, J. P., Klein, J., Chou, C., et al. (2013). A crossover pilot study evaluating the functional outcomes of two different types of robotic movement training in chronic stroke survivors using the arm exoskeleton bones. J. Neuroeng. Rehabil. 10, 112. doi:10.1186/1743-0003-10-112

Morales, R., Badesa, F. J., García-Aracil, N., Sabater, J. M., and Pérez-Vidal, C. (2011). Pneumatic robotic systems for upper limb rehabilitation. Med. Biol. Eng. Comput. 49, 1145-1156. doi:10.1007/s11517-011-0814-3

Mottram, C. J., Suresh, N. L., Heckman, C. J., Gorassini, M. A., and Rymer, W. Z. (2009). Origins of abnormal excitability in biceps brachii motoneurons of spastic-paretic stroke survivors. J. Neurophysiol. 102, 2026-2038. doi:10.1152/jn. 00151.2009

Moubarak, S., Pham, M. T., Moreau, R., and Redarce, T. (2010). "Gravity compensation of an upper extremity exoskeleton,” in 2010 Annual International Conference of the IEEE Engineering in Medicine and Biology Society (EMBC) (IEEE), 4489-4493.

Moubarak, S., Pham, M. T., Pajdla, T., and Redarce, T. (2009). "Design and modeling of an upper extremity exoskeleton," in World Congress on Medical Physics and Biomedical Engineering, September 7 - 12, 2009, eds O. Dïssel and W. C. Schlegel (Munich: Springer Berlin Heidelberg), 476-479.

Musampa, N. K., Mathieu, P. A., and Levin, M. F. (2007). Relationship between stretch reflex thresholds and voluntary arm muscle activation in patients with spasticity. Exp. Brain Res. 181, 579-593. doi:10.1007/s00221-007-0956-6

Nef, T., Mihelj, M., and Riener, R. (2007). Armin: a robot for patient-cooperative arm therapy. Med. Biol. Eng. Comput. 45, 887-900. doi:10.1007/s11517-007-0226-6

Nudo, R. J. (2013). Recovery after brain injury: mechanisms and principles. Front. Hum. Neurosci. 7:887. doi:10.3389/fnhum.2013.00887

Patton, J. L., and Mussa-Ivaldi, F. A. (2002). Robot assisted adaptative training: custom force fields for teaching movement patterns. IEEE Rev. Biomed. Eng. 51, 636-646. doi:10.1109/TBME.2003.821035

Pennycott, A., Wyss, D., Vallery, H., Klamroth-Marganska, V., and Riener, R. (2012). Towards more effective robotic gait training for stroke rehabilitation: a review. $J$. Neuroeng. Rehabil. 9, 65. doi:10.1186/1743-0003-9-65

Perry, J., Rosen, J., and Burns, S. (2007). Upper-limb powered exoskeleton design. IEEE/ASME Trans. Mechatron. 2, 408-417. doi:10.1109/TMECH.2007.901934

Perry, J. C., and Rosen, J. (2006). "Design of a 7 degree-of-freedom upperlimb powered exoskeleton," in The First IEEE/RAS-EMBS International Conference on Biomedical Robotics and Biomechatronics, 2006, BioRob 2006 (IEEE), 805-810.

Pignolo, L., Dolce, G., Basta, G., Lucca, L. F., Serra, S., and Sannita, W. G. (2012). "Upper limb rehabilitation after stroke: aramis a robo-mechatronic innovative approach and prototype," in 4th IEEE RAS \& EMBS International Conference on Biomedical Robotics and Biomechatronics (BioRob), 2012 (IEEE), 1410-1414.

Pons, J. L. (2008). Wearable Robots: Biomechatronic Exoskeletons, London: Wiley.

Prange, G. B., Jannink, M. J., Stienen, A. H., van der Kooij, H., MJ, I. J., and Hermens, H. J. (2010). An explorative, cross-sectional study into abnormal muscular coupling during reach in chronic stroke patients. J. Neuroeng. Rehabil. 7, 14. doi:10.1186/1743-0003-7-14

Rahman, M., Ouimet, T. K., Saad, M., Kenne, J.-P., and Archambault, P. (2010). "Development and control of a wearable robot for rehabilitation of elbow and shoulder joint movements," in IECON 2010 - 36th Annual Conference on IEEE Industrial Electronics Society (IEEE), 1506-1511.

Rahman, M., Rahman, M., Saad, M., Ochoa-Luna, C., Ferrer, S., and Archambault, P. (2013). Control of an Upper Extremity Exoskeleton Robot to Provide Active Assistive Therapy. 105-110.

Reinkensmeyer, D. J., Wolbrecht, E. T., Chan, V., Chou, C., Cramer, S. C., and Bobrow, J. E. (2012). Comparison of three-dimensional, assist-as-needed robotic arm/hand movement training provided with pneu-wrex to conventional tabletop therapy after chronic stroke. Am. J. Phys. Med. Rehabil. 91(11 Suppl. 3), S232-S241. doi:10.1097/PHM.0b013e31826bce79

Reisman, D. S., and Scholz, J. P. (2003). Aspects of joint coordination are preserved during pointing in persons with post-stroke hemiparesis. Brain 126, 2510-2527. doi:10.1093/brain/awg246

Reisman, D. S., and Scholz, J. P. (2006). Workspace location influences joint coordination during reaching in post-stroke hemiparesis. Exp. Brain Res. 170, 265-276. doi:10.1007/s00221-005-0209-5

Ren, Y., Kang, S. H., Park, H.-S., Wu, Y.-N., and Zhang, L.-Q. (2013). Developing a multi-joint upper limb exoskeleton robot for diagnosis, therapy, and outcome 
evaluation in neurorehabilitation. IEEE Trans. on Neural Syst. Rehabil. Eng. 21, 490-499. doi:10.1109/TNSRE.2012.2225073

Riener, R., Guidali, M., Keller, U., Duschau-Wicke, A., Klamroth, V., and Nef, T. (2011). Transferring Armin to the clinics and industry. Top. Spinal Cord Inj. Rehabil. 17, 54-59. doi:10.1310/sci1701-54

Roby-Brami, A., Feydy, A., Combeaud, M., Biryukova, E. V., Bussel, B., and Levin, M. F. (2003a). Motor compensation and recovery for reaching in stroke patients. Acta Neurol. Scand. 107, 369-381. doi:10.1034/j.1600-0404.2003.00021.x

Roby-Brami, A., Jacobs, S., Bennis, N., and Levin, M. F. (2003b). Hand orientation for grasping and arm joint rotation patterns in healthy subjects and hemiparetic stroke patients. Brain Res. 969, 217-229. doi:10.1016/S0006-8993(03)02334-5

Roh, J., Rymer, W. Z., Perreault, E. J., Yoo, S. B., and Beer, R. F. (2013). Alterations in upper limb muscle synergy structure in chronic stroke survivors. J. Neurophysiol. 109, 768-781. doi:10.1152/jn.00670.2012

Rosati, G., Gallina, P., Masiero, S., and Rossi, A. (2005). "Design of a New 5 dof WireBased Robot for Rehabilitation," in 9th International Conference on Rehabilitation Robotics, 2005. ICORR 2005 (IEEE), 430-433.

Sanchez, R. J. Jr., Wolbrecht, E., Smith, R., Liu, J., Rao, S., Cramer, S., et al. (2005). “A Pneumatic Robot for Re-Training Arm Movement After Stroke: Rationale and Mechanical Design," in 9th International Conference on Rehabilitation Robotics, 2005. ICORR 2005 (IEEE), 500-504.

Schiele, A. (2008). "An explicit model to predict and interpret constraint force creation in PHRI with exoskeletons," in IEEE International Conference on Robotics and Automation, 2008. ICRA 2008 (IEEE), 1324-1330.

Scott, S. H., and Winter, D. A. (1993). Biomechanical model of the human foot: kinematics and kinetics during the stance phase of walking. J. Biomech. 26, 1091-1104. doi:10.1016/S0021-9290(05)80008-9

Simkins, M., Kim, H., Abrams, G., Byl, N., and Rosen, J. (2013). "Robotic unilateral and bilateral upper-limb movement training for stroke survivors afflicted by chronic hemiparesis," in IEEE International Conference on Rehabilitation Robotics (ICORR), 2013 (IEEE), 1-6.

Song, Z., Guo, S., Pang, M., Zhang, S., Xiao, N., Gao, B., et al. (2014). Implementation of resistance training using an upper-limb exoskeleton rehabilitation device for elbow joint. J. Med. Biol. Eng. 34, 188-196. doi:10.5405/jmbe.1337

Stienen, A. H. A., Hekman, E. E. G., ter Braak, H., Aalsma, A. M. M., Van der Helm, F. C. T., and van der Kooij, H. (2008). Design of a Rotational Hydro-Elastic Actuator for an Active Upper-Extremity Rehabilitation Exoskeleton. 881-888.

Sukal, T. M., Ellis, M. D., and Dewald, J. P. (2007). Shoulder abduction-induced reductions in reaching work area following hemiparetic stroke: neuroscientific implications. Exp. Brain Res. 183, 215-223. doi:10.1007/s00221-007-1029-6

Sukal-Moulton, T., Krosschell, K. J., Gaebler-Spira, D. J., and Dewald, J. P. (2014). Motor impairment factors related to brain injury timing in early hemiparesis. Part I: expression of upper-extremity weakness. Neurorehabil. Neural Repair 28, 13-23. doi:10.1177/1545968313500564

Taub, E., Uswatte, G., Mark, V. W., and Morris, D. M. (2006). The learned nonuse phenomenon: implications for rehabilitation. Eura. Medicophys. 42, 241-256.

Ting, L. H. (2007). Dimensional reduction in sensorimotor systems: a framework for understanding muscle coordination of posture. Prog. Brain Res. 165, 299-321. doi:10.1016/S0079-6123(06)65019-X

Toth, A., Arz, G., Fazekas, G., Bratanov, D., and Zlatov, N. (2004). "25 Post stroke shoulder-elbow physiotherapy with industrial robots," in Advances in Rehabilitation Robotics (Heidelberg: Springer), 391-411.

Tropea, P., Monaco, V., Coscia, M., Posteraro, F., and Micera, S. (2013). Effects of early and intensive neuro-rehabilitative treatment on muscle synergies in acute post-stroke patients: a pilot study. J. Neuroeng. Rehabil. 10, 103. doi:10.1186/ 1743-0003-10-103

Trumbower, R. D., Ravichandran, V. J., Krutky, M. A., and Perreault, E. J. (2010). Contributions of altered stretch reflex coordination to arm impairments following stroke. J. Neurophysiol. 104, 3612-3624. doi:10.1152/jn.00804.2009

Tsagarakis, N. G., and Caldwell, D. G. (2003). Development and control of a "softactuated" exoskeleton for use in physiotherapy and training. Auton. Robots 15, 21-33. doi:10.1023/A:1024484615192

Tsai, B.-C., Wang, W. W., Hsu, L.-C., Fu, L. C., and Lai, J. (2010). "An articulated rehabilitation robot for upper limb physiotherapy and training," in IEEE/RSJ International Conference on Intelligent Robots and Systems (IROS), 2010 (IEEE), 1470-1475.

Twitchell, T. E. (1951). The restoration of motor function following hemiplegia in man. Brain 74, 443-480. doi:10.1093/brain/74.4.443
Van der Helm, F. V., Veeger, H., Pronk, G., der Woude, L. V., and Rozendal, R. (1992). Geometry parameters for musculoskeletal modelling of the shoulder system. $J$. Biomech. 25, 129-144. doi:10.1016/0021-9290(92)90270-B

van Kordelaar, J., van Wegen, E. E., and Kwakkel, G. (2012). Unraveling the interaction between pathological upper limb synergies and compensatory trunk movements during reach-to-grasp after stroke: a cross-sectional study. Exp. Brain Res. 221, 251-262. doi:10.1007/s00221-012-3169-6

van Kordelaar, J., van Wegen, E. E., Nijland, R. H., Daffertshofer, A., and Kwakkel, G. (2013). Understanding adaptive motor control of the paretic upper limb early poststroke: the explicit-stroke program. Neurorehabil. Neural Repair 27, 854-863. doi:10.1177/1545968313496327

Vertechy, R., Frisoli, A., Dettori, A., Solazzi, M., and Bergamasco, M. (2009). Development of a New Exoskeleton for Upper Limb Rehabilitation (IEEE), 188-193.

Volpe, B. T., Huerta, P. T., Zipse, J. L., Rykman, A., Edwards, D., Dipietro, L., et al. (2009). Robotic devices as therapeutic and diagnostic tools for stroke recovery. Arch. Neurol. 66, 1086-1090. doi:10.1001/archneurol.2009.182

Volpe, B. T., Lynch, D., Rykman-Berland, A., Ferraro, M., Galgano, M., Hogan, N., et al. (2008). Intensive sensorimotor arm training mediated by therapist or robot improves hemiparesis in patients with chronic stroke. Neurorehabil. Neural Repair 22, 305-310. doi:10.1177/1545968307311102

Wagner, J. M., Dromerick, A. W., Sahrmann, S. A., and Lang, C. E. (2007). Upper extremity muscle activation during recovery of reaching in subjects with poststroke hemiparesis. Clin. Neurophysiol. 118, 164-176. doi:10.1016/j.clinph.2006. 09.022

Wee, S. K., Hughes, A. M., Warner, M., and Burridge, J. H. (2014). Trunk restraint to promote upper extremity recovery in stroke patients: a systematic review and meta-analysis. Neurorehabil. Neural Repair 28, 660-677. doi:10.1177/ 1545968314521011

Wolbrecht, E., Chan, V., Reinkensmeyer, D., and Bobrow, J. (2008). Optimizing compliant, model-based robotic assistance to promote neurorehabilitation. IEEE Trans. Neural Syst. Rehabil. Eng. 16, 286-297. doi:10.1109/TNSRE.2008.918389

Wolf, S. L., Winstein, C. J., Miller, J. P., Taub, E., Uswatte, G., Morris, D., et al. (2006). Effect of constraint-induced movement therapy on upper extremity function 3 to 9 months after stroke: the excite randomized clinical trial. JAMA 296, 2095-2104. doi:10.1001/jama.296.17.2095

Yu, W., and Rosen, J. (2013). Neural PID control of robot manipulators with application to an upper limb exoskeleton. IEEE Trans. Cybern. 43, 673-684. doi:10.1109/TSMCB.2012.2214381

Yu, W., Rosen, J., and Li, X. (2011). "PID admittance control for an upper limb exoskeleton," in American Control Conference (ACC), 2011 (IEEE), 1124-1129.

Zackowski, K. M., Dromerick, A. W., Sahrmann, S. A., Thach, W. T., and Bastian, A. J. (2004). How do strength, sensation, spasticity and joint individuation relate to the reaching deficits of people with chronic hemiparesis? Brain 127(Pt 5), 1035-1046. doi:10.1093/brain/awh116

Zhang, H., Austin, H., Buchanan, S., Herman, R., Koeneman, J., and He, J. (2011). "Feasibility study of robot-assisted stroke rehabilitation at home using Rupert," in International Conference on Complex Medical Engineering (CME), 2011 IEEE/ICME (IEEE), 604-609.

Zhang, L.-Q., Park, H.-S., and Ren, Y. (2007). "Developing an intelligent robotic arm for stroke rehabilitation," in IEEE 10th International Conference on Rehabilitation Robotics (IEEE), 984-993.

Conflict of Interest Statement: The authors declare that the research was conducted in the absence of any commercial or financial relationships that could be construed as a potential conflict of interest.

Received: 30 July 2014; accepted: 06 November 2014; published online: 01 December 2014.

Citation: Jarrassé N, Proietti T, Crocher V, Robertson J, Sahbani A, Morel G and Roby-Brami A (2014) Robotic exoskeletons: a perspective for the rehabilitation of arm coordination in stroke patients. Front. Hum. Neurosci. 8:947. doi: 10.3389/fnhum.2014.00947

This article was submitted to the journal Frontiers in Human Neuroscience. Copyright (c) 2014 Jarrassé, Proietti, Crocher, Robertson, Sahbani, Morel and RobyBrami. This is an open-access article distributed under the terms of the Creative Commons Attribution License (CC BY). The use, distribution or reproduction in other forums is permitted, provided the original author(s) or licensor are credited and that the original publication in this journal is cited, in accordance with accepted academic practice. No use, distribution or reproduction is permitted which does not comply with these terms. 